\title{
Best Paper Award
}

Der Beitrag «Werbung mit Knappheitssignalen: Die Rolle des Typs des Signals, der Produktkategorie und der Sachargumente» von Heribert Gierl verdient den «Best Paper Award 2010» von «Die Unternehmung» aus folgenden Gründen: Erstens wird im Bereich der Verhaltensforschung ein höchst relevantes Thema für die Wissenschaft und für Unternehmen behandelt. Zweitens sind der Untersuchungsgegenstand und der Versuchsaufbau innovativ und drittens verbindet Heribert Gierl mit seinem Beitrag Praxis und Theorie, indem theoretische Erkenntnisse empirisch überprüft und auf praxisrelevante Fragestellungen angewandt werden.

Relevanz des Themas: Unternehmen werben zunehmend mit Zusätzen, wie z.B. «nur solange der Vorrat reicht» oder «Limited Edition». Dabei unterstellen sowohl Hersteller als auch Händler, dass Produkte, die aus Konsumentensicht knapp erscheinen, für Verbraucher erstrebenswerter werden. Der Beitrag von Heribert Gierl untersucht genau diesen Sachverhalt unter Berücksichtigung verschiedener Einflussfaktoren. Knappheit von Produkten basiert entweder auf einer hohen Nachfrage oder auf einer geringen Angebotsmenge. Für Hersteller und Händler stellt sich daher zum einen die Frage, ob diese beiden Typen von Knappheit unterschiedliche Wirkungen auf Konsumenten haben. Zum anderen ist der Effekt von Knappheit auf das Konsumentenverhalten gegebenenfalls von Eigenschaften der Produkte oder von der aus Konsumentensicht wahrgenommenen Produktqualität abhängig. Im Rahmen der Eigenschaften der Produkte wird vor allem deren Eignung zur Befriedigung sozialer Bedürfnisse, wie z.B. Anerkennung und Bewunderung in die Untersuchung mit einbezogen. Hierzu differenziert der Autor zwischen sozial sichtbaren Produkten und solchen, die nicht öffentlich genutzt oder verbraucht werden. Die wahrgenommene Produktqualität wird über die Lieferung starker Sachargumente für ein Produkt durch Unternehmen abgebildet. Die Betrachtung der drei Einflussfaktoren liefert wesentliche Erkenntnisse für die Einstellung von Konsumenten gegenüber knappen Produkten sowie das daraus resultierende Konsumentenverhalten und lässt die Ableitung von erfolgsrelevanten Handlungsimplikationen für die Praxis zu.

Innovativer Untersuchungsgegenstand und Versuchsaufbau: Der Beitrag ist in mehrfacher Hinsicht innovativ. Obwohl einige Arbeiten nahe Forschungsbereiche, wie z.B. Limitierung von Promotions oder Knappheit von Incentives untersucht haben, ist bis dato eine detaillierte Betrachtung der Auswirkungen wahrgenommener Knappheit auf das Konsumentenverhalten offen geblieben. Dies gilt insbesondere für die zusätzliche Differenzierung zwischen nachfrage- und unternehmensbedingter Knappheit sowie die Berücksichtigung von Produkteigenschaften sowie Qualitätswahrnehmung als weitere Einflussfaktoren. Darüber hinaus ist die empirische Vorgehensweise von Heribert Gierl anhand eines experimentellen Forschungsdesigns als besonders geeignet für die Beantwortung der vorliegenden Fragestellung einzustufen.

Verbindung von Theorie und Praxis: In dem vorliegenden Beitrag werden zunächst anhand der Reaktanz-Theorie, der Commodity-Theorie sowie den Theorien zu Conspicuous Consumption das wissenschaftliche Fundament geschaffen. Darauf aufbauend untersucht Gierl 
praxisrelevante Fragestellungen und leitet nachvollziehbar wesentliche Implikationen für die Praxis ab. Der Autor erarbeitet dementsprechend Erkenntnisse in wissenschaftlich präziser und fundierter Form und präsentiert die Ergebnisse zugleich systematisch und gut strukturiert. Ein sehr gelungener Beitrag.

Manfred Bruhn, Universität Basel

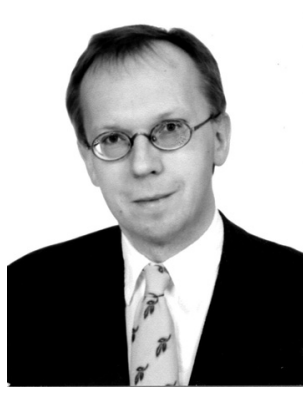

Heribert Gierl, Prof. Dr., unterrichtet seit 1992 an der Wirtschaftswissenschaftlichen Fakultät der Universität Augsburg Betriebswirtschaftslehre mit dem Schwerpunkt Marketing. Zuvor vertrat er eine Professur für Marktlehre an der Fakultät für Landwirtschaft und Gartenbau an der TU München, eine Professur für Marketing an der Universität Regensburg, wo er bei Professor Böcker promoviert und sich habilitiert hatte, und eine Gründungsprofessur für Betriebswirtschaftslehre an der Bergakademie Freiberg. Er hatte Rufe an die TU München, an die Bergakademie Freiberg und an die Universität zu Köln. In der Lehre bietet er mit seinem Lehrstuhlteam und Gastdozenten aus der Praxis Kurse aus den drei Spezialisierungsbereichen Marketing Research, Marketing Management und Consumer Behavior an. Insofern erhalten seine Studenten eine branchenübergreifende und berufsorientierte Ausbildung im Bereich der quantitativen und qualitativen Methoden der Marktforschung, im Bereich des strategischen und operativen Marketings und im Bereich der psychologisch fundierten Techniken, wie sich Zielgruppen eines Unternehmens beeinflussen lassen. Er ist Vorstand der WIMU e.V., dessen Aufgabe darin besteht, Beispiele der unternehmerischen Praxis in die studentische Ausbildung zu integrieren. Seine Forschungsschwerpunkte liegen in der Bereichen des Industriegütermarketings, der deskriptiven Entscheidungstheorie und der Konsumgüterwerbung. Einen besonderen Wert liegt er auf die akademische Qualifikation von Doktoranden und die Weiterqualifikation von Habilitanden, in deren Kooperation Publikationen in international angesehenen Zeitschriften seines Fachs entstehen.

\section{Reviewer Award}

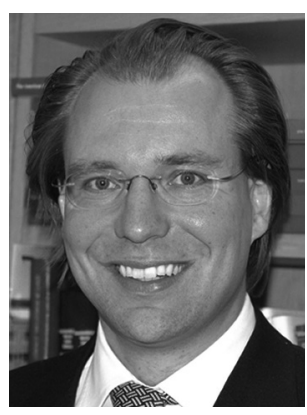

Alexander F. Wagner, Ph.D., ist Swiss Finance Institute Assistenzprofessor für Finance an der Universität Zürich. Vor seinem Ruf nach Zürich studierte und forschte er fünf Jahre lang an der Harvard Universität, wo er seinen Ph.D. erwarb. Professor Wagner forscht und lehrt in den Bereichen Corporate Finance, Corporate Governance und Behavioral Economics and Finance. Seine Arbeiten sind in Zeitschriften wie dem Journal of Economic History, dem Journal of the European Economic Association und dem Journal of Financial Intermediation erschienen. Er erhielt verschiedene Auszeichnungen, darunter den Ehrenring der Republik Österreich sowie das APARTStipendium der Österreichischen Akademie der Wissenschaften. 